\title{
Mu2e-II Injection from PIP-II
}

\author{
David Neuffer \\ Fermilab, PO Box 500, Batavia IL 60510 USA
}

\begin{abstract}
We discuss injection of $800 \mathrm{MeV}$ proton beam from PIP-II into the production target for Mu2e-II, assuming a targeting and $\mu$ production scenario similar to mu2e. The incoming beam trajectory must be modified from the mu2e parameters to match the focusing fields. Adding a vertical deflection at injection enables the injected beam to reach the target. Other differences from the mu2e system must be considered, including changes in the target structure, the radiation shielding and beam dump/absorber. $\mathrm{H}^{-}$beam should be stripped to $\mathrm{p}^{+}$. Other variations are discussed.
\end{abstract}

\section{INTRODUCTION}

The PIP-II project will provide a $800 \mathrm{MeV}$ proton beam with cw capability, with beam power up to the MW level available for user experiments.[1] An upgraded or extended version of the Fermilab mu2e experiment[2,3], labeled mu2e-II, has been identified as a primary future user of that beam.[4] The mu2e experiment will initially use $8 \mathrm{GeV}$ proton beam from a compact bunch stored in the Delivery Ring, extracted by slow extraction, and is limited in intensity to $<10 \mathrm{~kW}$. The PIP-II beam could instead provide more than $100 \mathrm{~kW}$ of $800 \mathrm{MeV}$ beam directly from the linac, where the linac beam can be formed into timing sequences that can match mu2e experimental requirements very precisely. The mu2e collaboration has expressed interest in an extension to the mu2e experiment based on the PIP-II linac. [5]

Our initial framework for mu2e-II is to begin with the mu2e Detector layout, but inserting $800 \mathrm{MeV}$ beam in place of the $8 \mathrm{GeV}$ beam, and reusing as much of the initial experiment infrastructure as practical. Fig. 1 shows that detector, with the proton beam injection indicated by an arrow. The proton beam is injected off-center directed toward a production target in the production solenoid (PS). Low-energy backward-produced pions and muons are directed toward a transport solenoid (TS). The transport and associated collimators filter the resulting beam into low-energy $\mu^{-}$that are stopped in the target, and captured in atomic orbits. Electrons produced from $\mu$-decay and $\mu \rightarrow \mathrm{e}$ conversion events are observed in the detector solenoid (DS) tracker; $\mu \rightarrow \mathrm{e}$ conversion is identified by a monoenergetic $105 \mathrm{MeV}$ electron.

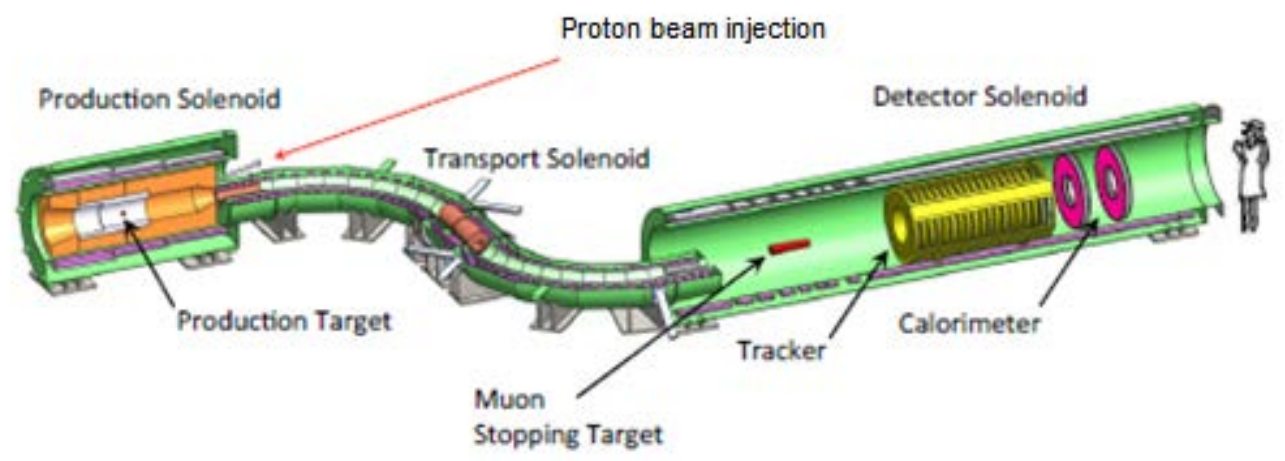

FIGURE 1. The mu2e Detector. Proton beam is injected off-axis toward the production target, producing secondaries that are transported to the $\mathrm{Al}$ stopping target. $\mu \rightarrow$ e conversion events are detected as $105 \mathrm{MeV}$ electrons in the tracker/calorimeter.

\section{Delivery of beam from the PIP-II Linac}

Fig. 2 displays the PIP-II linac as it will be constructed on the Fermilab site, with the beamlines to the Booster and mu2e indicated. A. Vivoli has designed beamline lattices for this geometry.[6] Lattice functions and elements for this lattice are displayed in figure 3. The transport to mu2e-II splits from the Booster line and is directed into the beamline from the Delivery Ring into the mu2e experiment. (After this transfer point, the lattice is the same as that initially planned for mu2e $8 \mathrm{GeV}$ injection, and includes beam optics and collimators used for the mu2e extinction system, wnich could be included in the mu2e-II system.)

For compatibility with $\mathrm{H}^{-}$injection into the Booster, the linac accelerates $\mathrm{H}^{-}$ions, and the bending fields are limited to $0.25 \mathrm{~T}$ to avoid magnetic stripping to $\mathrm{H}^{0}$. $\mathrm{H}^{-}$ions would therefore be sent down the mu2e-II line. However 
the production solenoid (PS) has fields up to $\sim 4 \mathrm{~T}$ before the target. At an injection angle of $13.6^{\circ}$, this implies $B_{t}=\sim 0.9 \mathrm{~T}$ perpendicular to the beam. The stripping time can be estimated using the formula of Schrek:

$$
\tau=\frac{a}{3.197 B_{t} p} \exp \left(\frac{b}{3.197 B_{t} p}\right) \quad \text { seconds, }
$$

$p=1.463 \mathrm{GeV} / \mathrm{c}$ for $800 \mathrm{MeV} \mathrm{H}^{-}$and $B_{t}=0.9 \mathrm{~T}$, and $a$ and $b$ are parameters fitted from data. [7,8] Keating et al. obtained $a=3.07310^{-14}$ and $b=44.14$ from $800 \mathrm{MeV}$ data. [9] At these parameters $\tau=2.6210^{-10} \mathrm{~s}$ or $(\mathrm{c} \tau=8 \mathrm{~cm})$. Thus much of the $\mathrm{H}^{-}$would strip to $\mathrm{H}^{0}$ within the magnet, and non-interacting $\mathrm{H}^{-}$and $\mathrm{H}^{0}$ would strip to $\mathrm{p}\left(\mathrm{H}^{+}\right)$in the target.

The mixture of charge states in the PS would make the beam transport to and past the target difficult to control. It would therefore be desirable to strip the linac $\mathrm{H}^{-}$beam in the mu2e-II transport upstream of the production target so that only proton beam reaches the PS. This stripper should be downstream of the separation from the Booster line and possibly upstream of the bend into the mu2e line. (The bend would separate out unstripped $\mathrm{H}^{-}$and $\mathrm{H}^{0}$.) The stripper would be a thin carbon or diamond foil, similar to that used for $\mathrm{H}^{-}$injection into the Booster or that used at SNS for $800 \mathrm{MeV} \mathrm{H}^{-}$injection.

A potential advantage of the stripper is that it could be incorporated into an extinction system. [10] A beam bump onto and off the stripper foil could be timed to match the bunch train window for occupied beam. Any downstream bending magnet would then separate out mistimed beam, providing another level of extinction. The original mu2e extinction system may not then be needed. The mu2e-II beam line should be reoptimized to incorporate these changes.

The $\mathrm{H}^{-}$stripping time is a strong function of the magnetic field and could possibly be avoided in a significantly different beam injection scheme (injection closer to axis or along the axis, or a lower field solenoid). For example, reducing the transverse field by a factor of 2 increases $c \tau$ to $5.8 \mathrm{~km}$. The other possible advantages of earlier stripping would remain, and it would still be preferable.

One could also consider magnetic stripping of $\mathrm{H}^{-} \rightarrow \mathrm{H}^{0}$ just before entering the PS. The beam from stripper to target would then be undeflected. An undesirable spread in deflection due to varying stripping location would be introduced ( $\sim 1 \mathrm{mrad}$ or more) as well as an average deflection from the stripping magnet. Complications from stripping inefficiency in the PS area and reduced beam control (no steering or focusing after stripping) would exist. This variant in stripping geometry could also be studied in more detail. 


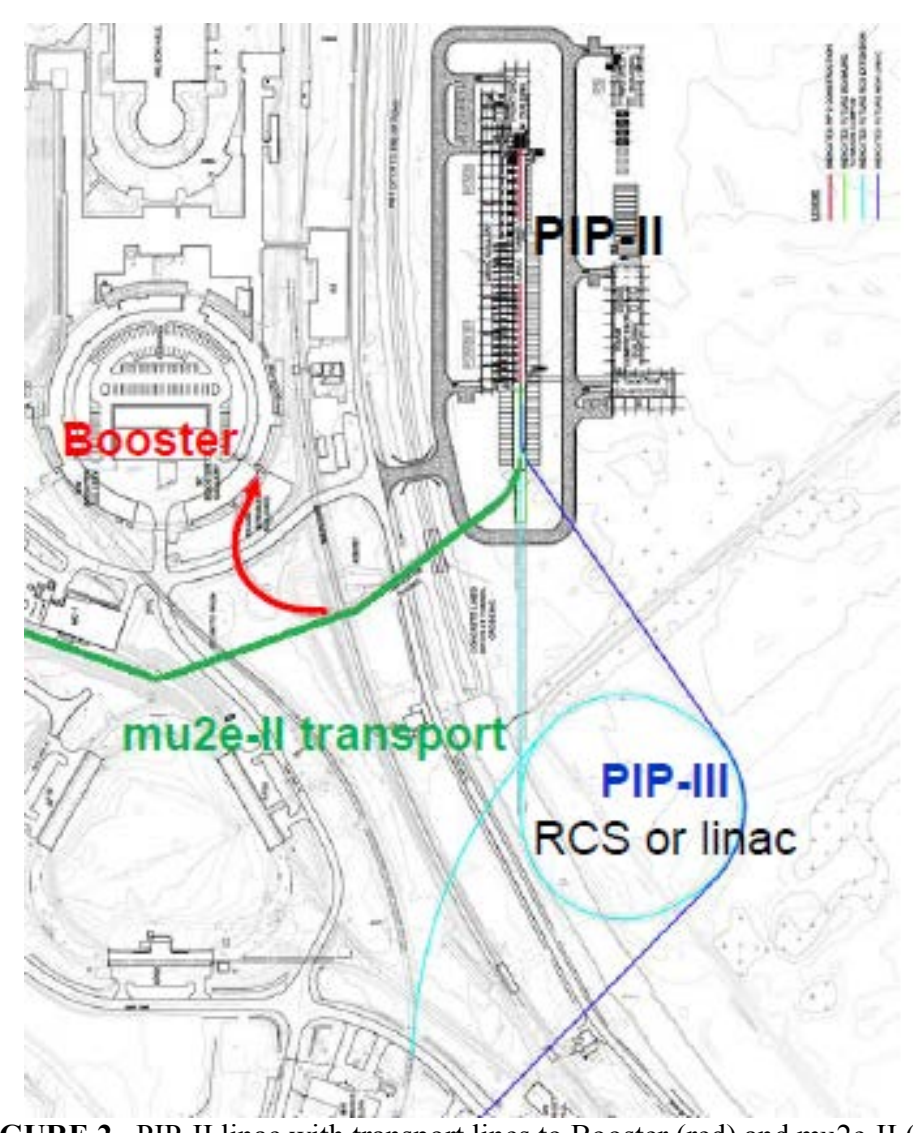

FIGURE 2. PIP-II linac with transport lines to Booster (red) and mu2e-II (green) indicated.

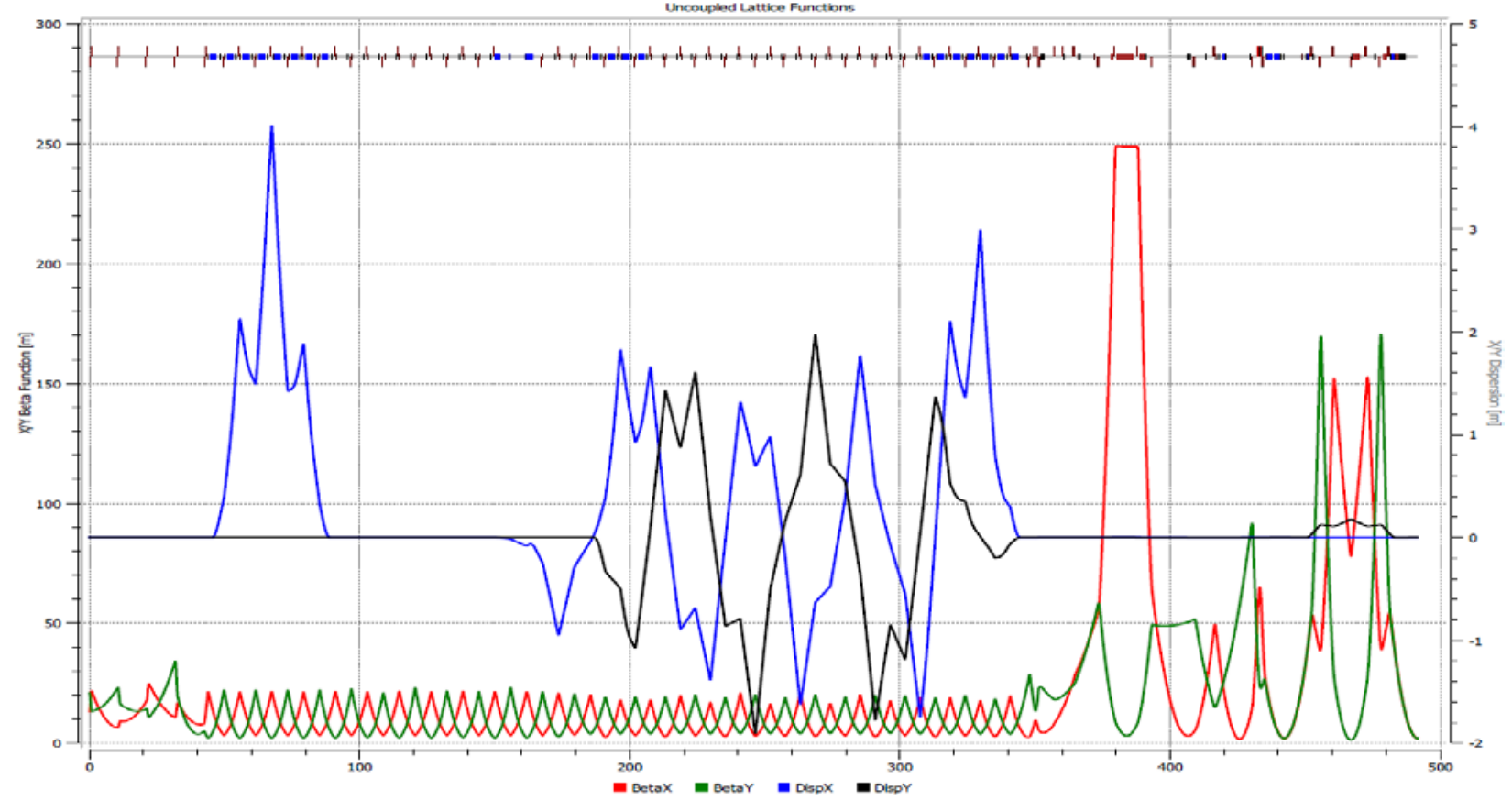

FIGURE 3. Lattice functions for the transport from the PIP-II linac into the mu2e enclosure. 


\section{Bunch train formation for mu2e-II}

The PIP-II linac originates from a $162.5 \mathrm{MHz}$ rfq and includes a chopper system that can produce an arbitrary pattern of filled or empty $162.5 \mathrm{MHz}$ buckets. The maximum current per bucket is $10 \mathrm{ma}\left(3.85 \cdot 10^{8}\right.$ particles $)$, although a more conservative limit of $5 \mathrm{ma}$ is better suited to stable operation. The average current is limited to $2 \mathrm{ma}$ $\left(7.7 \cdot 10^{7}\right.$ particles/bunch). The PIP-II timeline must reserve time for the pulsed injection into the $20 \mathrm{~Hz}$ Booster, this requires $\sim 3 \mathrm{~ms}$ out of every $50 \mathrm{~ms}$. The desired mu2e spill is a relatively short beam spill followed by a gap matched to the muon lifetime in the stopping target. Figure 4 shows a bunch spill pattern for mu2e-II modelled on the initial mu2e plan, and designed to provide $\sim 100 \mathrm{~kW}$ of beam on target. The time between bunch spills is $1.6 \mu$ s (similar to the Delivery Ring period of $1.695 \mu \mathrm{s}$ ). Only $75 \mathrm{ma}$ buckets are required in each spill; the resulting beam pulse is $\sim 43 \mathrm{~ns}$. This is much shorter than the $\sim 250 \mathrm{~ns}$ of the beam spill / turn for mu2e. This should provide a cleaner separation between primary beam arrival and the later captured $\mu$ decay.

An important advantage of the PIP-II source is that spill to spill uniformity should be much better for mu2e. mu2e uses slow extractionfrom the Delivery Ring, which has very large spill to spill variation. The PIP-II source is also not tied to the $1.6 \mu \mathrm{s}$ cycle. For other stopping target materials, the captured $\mu$ lifetime is much less, and a shorter cycle time may provide a better data distribution.

Another advantage of the PIP-II beam is its small emittance. The rms normalized emittance of the beam is $0.3 \mathrm{~mm}$-mrad. This is an order of magnitude less than that of the mu2e $8 \mathrm{GeV}$ beam. The geometric emittances are a factor of $\beta \gamma$ smaller; the geometric emittance of the PIP II beam is $\sim 0.2 \mathrm{~mm}-\mathrm{mrad}$, a bit smaller than the the mu2e beam at $\sim 0.35 \mathrm{~mm}$-mrad. Therefore it does not have to be focused as tightly into the production solenoid.

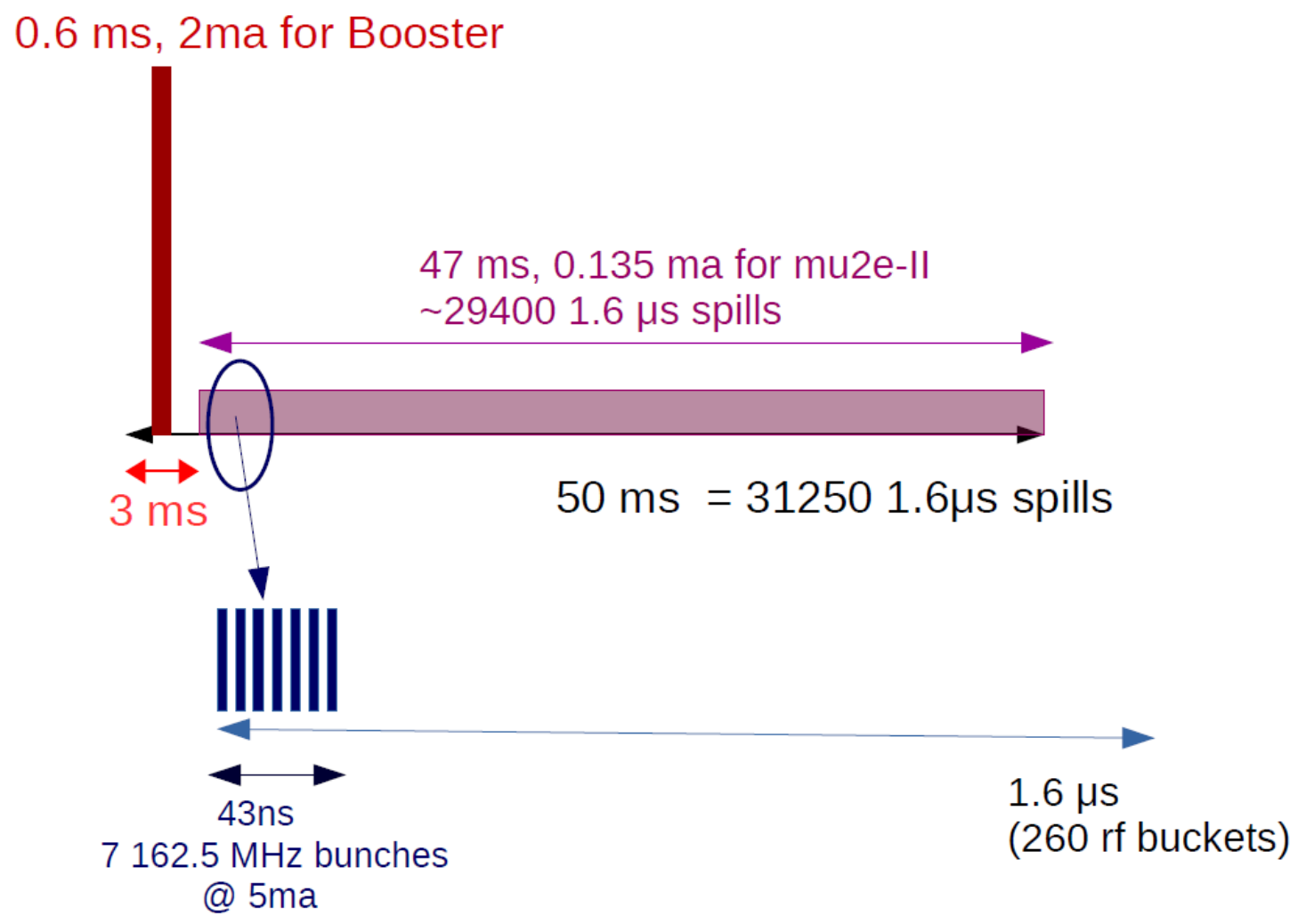

FIGURE 4. Possible time line for $100 \mathrm{~kW}$ mu2e-II operation of the PIP-II linac with a $1600 \mathrm{~ns}$ spill spacing, mixed with concurrent $20 \mathrm{~Hz}$ Booster injection.

\section{BEAM INJECTION INTO THE PRODUCTION SOLENOID}

The proton beam is injected into a production solenoid (PS) with relatively high solenoidal fields. $8 \mathrm{GeV}$ protons are relatively undeflected by the fields, but $800 \mathrm{MeV}$ protons are greatly deflected by the magnetic fields. Fig. 5 
shows the injection line into the PS. Beam enters at an $\sim 14^{\circ}$ angle (horizontally) and is off-center by $\sim 0.57 \mathrm{~m}$, aimed toward the target $\sim 2.35 \mathrm{~m}$ into the solenoid. [11] The beam follows a fairly narrow beampipe through the shielding within the PS toward the target. Non-interacting beam continues past the target into a dedicated dump. The radiation shield has an inner radius of $\sim 20 \mathrm{~cm}$, which opens to a larger radius downstream, so that the continuing beam and forward produced secondaries pass outside the solenoid into a downstream beam dump. [12]

The magnetic field is not constant in the PS; the axial field increases from $2.5 \mathrm{~T}$ to $\sim 4 \mathrm{~T}$ at the target (roughly linear with distance) and to a peak of $4.5 \mathrm{~T} \sim 1 / 2 \mathrm{~m}$ downstream of the target. The equations of motion within the solenoidal fields are:

$$
\begin{aligned}
& x^{\prime \prime}-S(s) y^{\prime}-\frac{1}{2} S^{\prime}(s) y=0 \\
& y^{\prime \prime}+S(s) x^{\prime}+\frac{1}{2} S^{\prime}(s) x=0
\end{aligned}
$$

through second order, where $S=B / B \rho$ and $B(s)$ is the solenoid field strength along the longitudinal coordinate $s$ and $B \rho=p / e$ is the magnetic rigidity of the protons (4.88 T-m for $800 \mathrm{MeV}$ protons). [13]

To third order, the equations are:

$$
\begin{aligned}
& x^{\prime \prime}=+S y^{\prime}+\frac{1}{2} S^{\prime} y+\frac{1}{4}\left(2 x^{\prime 2} y^{\prime} S+x^{\prime 2} y S^{\prime}+2 y^{\prime 3} S+y y^{\prime 2} S^{\prime}\right) \\
& y^{\prime \prime}=-S x^{\prime}-\frac{1}{2} S^{\prime} x-\frac{1}{4}\left(2 x^{\prime} y^{\prime 2} S+x y^{\prime 2} S^{\prime}+2 x^{\prime 3} S+x x^{\prime 2} S^{\prime}\right)
\end{aligned}
$$

For $8 \mathrm{GeV}$ beam a small vertical angle $\left(\sim 1.4^{\circ}\right)$ must be introduced to precisely hit the target center. The beam deflection from a straight track is $<2 \mathrm{~cm}$ before the target. Downstream of the target the deflection does grow to $\sim 8 \mathrm{~cm}$ at the solenoid exit.

$800 \mathrm{MeV}$ beam introduced at the same angle would be displaced from the target by about $30 \mathrm{~cm}$. It would be significantly deflected from the initial trajectory from about $1.25 \mathrm{~m}$ downstream of the entrance and hit the magnet heat and radiation shield (HRS). The trajectory would intersect the HRS further downstream and exit the PS at a radius of $\sim 0.6 \mathrm{~m}$.

A trajectory that is more appropriately matched to the PS geometry is determined by requiring it to pass through both the entry point and the target. To satisfy these constraints it must enter the solenoid at a different angle than the baseline: at an angle of $11.4^{\circ}$ horizontally and $8.3^{\circ}$ vertically. It would then deviate vertically by $\sim 10 \mathrm{~cm}$ halfway between the entry and the target, which is larger than the $4 \mathrm{~cm}$ radius of the mu2e HRS injection beampipe, as currently designed. It would exit the target at a different angle than the baseline, exiting the solenoid with a vertical displacement of $36 \mathrm{~cm}$ and angle of $\sim 12^{\circ}$. Thus the beam dump for noninteracting $800 \mathrm{MeV}$ beam following the PS would be displaced from that used for $8 \mathrm{GeV}$ beam.

Trajectories for these beam conditions have been calculated using a Mathematica-based model. Table 1 summarizes calculation results and fig. 6 displays some trajectory projections. This more matched solution could be adapted to a mu2e-like geometry, but the HRS geometry would have to be modified to match the beam trajectory. 


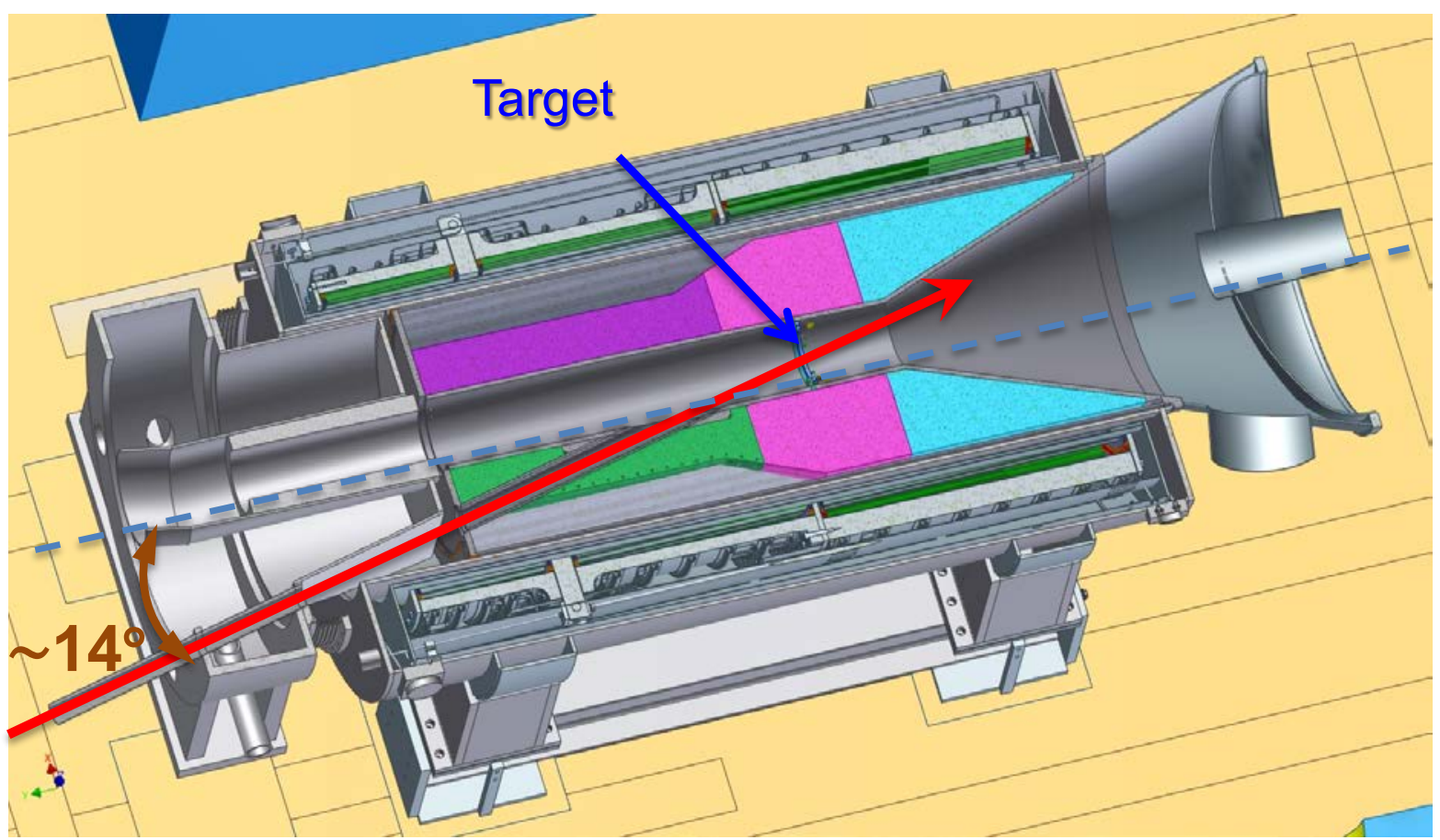

FIGURE 5. Entry of proton beam into the production solenoid. (from S. Werkema [11]). The bronze HRS is indicated by the green, purple, violet and cyan segments.

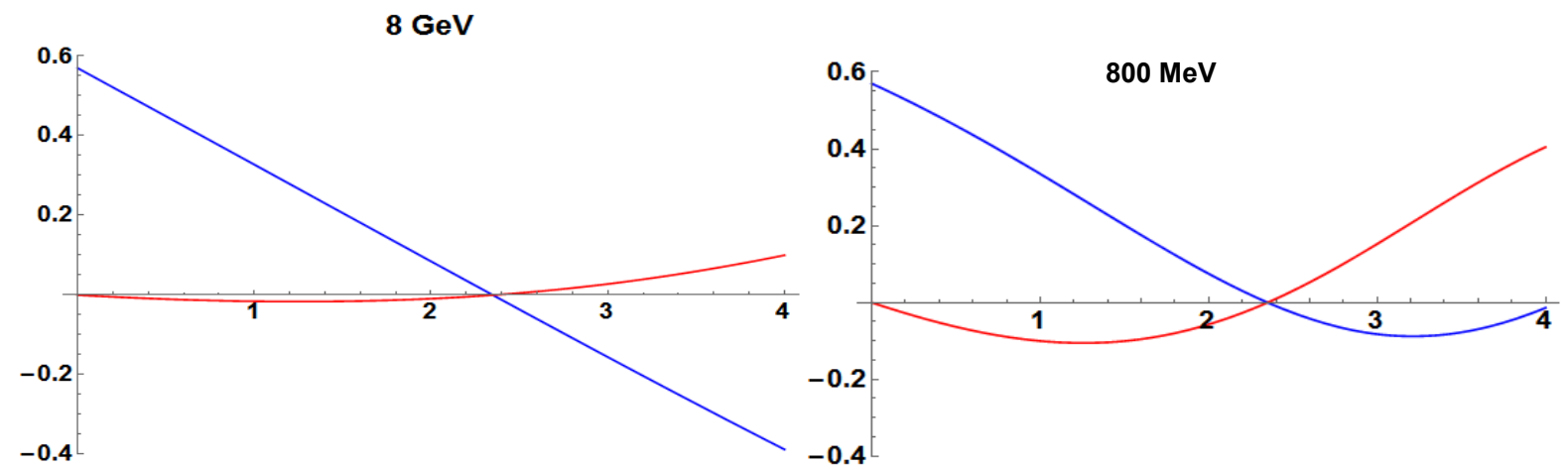

FIGURE 6. $x-y$ coordinates through PS for $8 \mathrm{GeV}$ protons (left) and $0.8 \mathrm{GeV}$ protons (right). Target is at $\mathrm{s}=2.35 \mathrm{~m}$

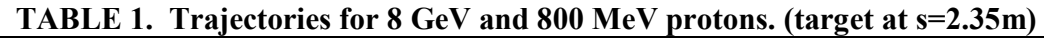

\begin{tabular}{lcccc}
\hline Position & $\mathbf{8 ~ G e V ~}(\mathbf{x}, \mathbf{y})$ & $\mathbf{8 ~ G e V}\left(\mathbf{x}^{\prime}, \mathbf{y}^{\prime}\right)$ & $\mathbf{8 0 0} \mathbf{M e V}(\mathbf{x}, \mathbf{y})$ & $\mathbf{8 0 0 ~ M e V ~}\left(\mathbf{x}^{\prime}, \mathbf{y}^{\prime}\right)$ \\
\hline $\mathrm{s}=0$ entrance & $0.57,0.0 \mathrm{~m}$ & $-13.6^{\circ},-1.4^{\mathrm{o}}$ & $0.57,0.0$ & $-11.4,-8.3^{\mathrm{o}}$ \\
$\mathrm{s}=2.35$ target & $0.0,0.0 \mathrm{~m}$ & $-13.6^{\circ}, 1.8^{\mathrm{o}}$ & $0.0,0.0$ & $-9.6^{\circ}, 10.9^{\mathrm{o}}$ \\
$\mathrm{s}=4 \mathrm{~m}$ exit & $-0.39,0.08 \mathrm{~m}$ & $-12.7^{\circ}, 5.1^{\mathrm{o}}$ & $-0.01,0.40$ & $10.2^{\mathrm{o}}, 11.4^{\mathrm{o}}$ \\
\hline
\end{tabular}

The $\sim$ cylindrical mu2e HRS lines the PS, with an inner radius of $\sim 20 \mathrm{~cm}$, flaring out to larger inner radius at the proton beam exit end. The HRS is mostly composed of bronze and it is designed to absorb the secondary particles and associated energy radiation from the target, protecting the superconducting coils. An $8 \mathrm{~cm}$ diameter cylindrical hole for the injected beam is drilled through the HRS. This hole would not accommodate the orbit of injected 800 $\mathrm{MeV}$ beam, which would deviate by $10 \mathrm{~cm}$ vertically from the center line. It would not be difficult in principle to modify the entry hole to accommodate that beam, with a redesign. The $8 \mathrm{GeV}$ beam itself has a full width of $\sim 1 \mathrm{~cm}$ and the $800 \mathrm{MeV}$ beam would be a bit smaller, because of its smaller emittance. 
A mu2e-II production solenoid would also need a HRS. The order of magnitude larger total power transmitted in mu2e-II would probably require a greater HRS, which could have a denser material (bronze $\rightarrow$ tungsten?) and may need to be thicker. The inner radius could be reduced a bit (with some loss in transmitted $\mu$ ), and an increase in the outer radius implies an increase in the PS coil diameter. More detailed energy deposition studies would be needed to determine the actual parameters needed for the mu2e-II upgrade.

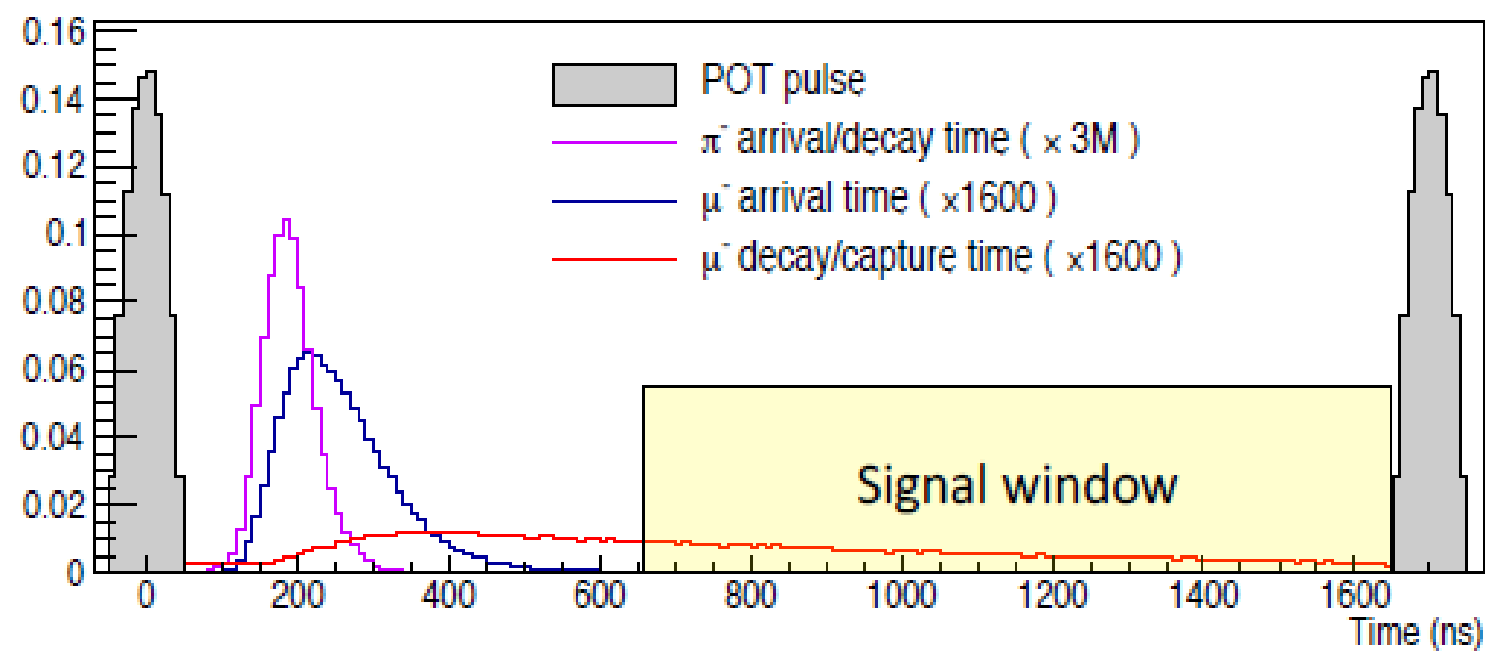

FIGURE 7. Timing plot for $\pi / \mu$ arrival and decay at the mu2e experiment with an initial proton on target (POT) width of 100ns. [4] Because of the large width in $\pi / \mu$ arrival times on the stopping target, the background-free signal window is not greatly increased by a shorter POT width; however, the background tails of distributions should be somewhat improved.

\section{R\&D REQUIREMENTS AND VARIATIONS}

This outline discussion indicates a straightforward extension of the mu2e experiment could be implemented, using the PIP-II beam. This potential injection mode for Mu2e-II will need further R\&D to be included in the mu2eII letter of intent. This includes:

- The PIP-II to mu2e transport needs to be updated. It should include a stripping foil with optics matched to positive protons downstream, with some provision to remove unstripped beam. A beam bump system to enhance extinction should be considered, and compared with use of the mu2e extinction system, adapted to the PIP-II beam. If bumping the beam off the stripper is preferable, then the final beam optics should be reoptimized. The final transport into the production solenoid should be rematched, including adding a vertical kick into a matched orbit for injection into the mu2e-II production solenoid, and any other modifications of the beam transport that may be needed.

$\bullet$

- The production target will need some redesign. The mu2e target is a $3 \mathrm{~mm}$ radius $16 \mathrm{~cm}$ long titanium cylinder suspended in the PS without an active cooling system, and would not survive $100 \mathrm{~kW}$ of beam. An actively cooled target will probably be needed. The additional radiation load on the target and a reoptimization of secondary $\pi-\mu$ production for the $800 \mathrm{MeV}$ beam may lead to a substantially different configuration.

- The heat and radiation shield (HRS) within the production solenoid (PS) must be updated to provide protection for the PS coils from the order of magnitude larger energy deposited from the target, as well as the differing beam entrance and exit paths. To first order, this can be done by replacing the bronze HRS with higher density $\mathrm{W}$ material. A first calculation indicates the radiation and the heat load on the PS coils would still be double that of mu2e $(8 \mathrm{~kW}$ beam $\rightarrow 100 \mathrm{~kW})$. [14] More work is needed to determine whether this is acceptable and to integrate it into a mu2e-II design.

- The mu2e target vault has a proton absorber for forward unstopped $8 \mathrm{GeV}$ primary beam. In the scenario presented here the $0.8 \mathrm{GeV}$ primary beam would exit in a different direction (vertically rather than horizontally) 
and would miss the proton absorber (PA). A new proton absorber might be needed. One could add a steering magnet after the production solenoid to direct these primaries to the PA. Or the direction and location of injection could be changed to match the PA location. (Vertical injection would lead to $\sim$ horizontal exit, which could be matched to the PA.) The higher beam power and lower beam energy might require a larger redesign of the proton absorber, however. Multiple scattering of protons in the target will spread the exiting beam over a much larger angle at $800 \mathrm{MeV}$. For $16 \mathrm{~cm} \mathrm{W,} \theta_{\mathrm{o}}=3.6^{\circ}$.

- $\quad$ Radiation deposition with $100 \mathrm{~kW} 800 \mathrm{MeV}$ p beam on target will be much larger and very different from that of $8 \mathrm{~kW} 8 \mathrm{GeV}$ beam. The beam dump and secondary production deposition will also be different, and a major effort in recalculation and redesign will be needed for the entire experimental area.

\section{Variations}

The somewhat different properties of the mu2e-II beam source allow consideration of significant variations from this extrapolation of the baseline mu2e approach.

The stopping target is $\mathrm{Al}$ and the mu2e beam pulse timing is matched to the mu decay lifetime in Al. Physics considerations may mandate the use of other targets such as titanium or a high-Z target like gold (Au) to explore the Z-dependence of mu2e conversion, and the beam pulse can be retailored to match.

The mu2e transport design, with the S-shaped solenoid, is designed to restrict mu acceptance to very low momenta, but it delivers beam onto the stopping target within a very broad time range. For tighter timing and broader acceptance, a significantly different transport geometry and stopping target configuration could be explored.

Since the PIP-2 beam will not produce antiprotons or other GeV-scale secondaries, the collimation and shielding geometries could be changed. One could consider forward produced muons, which could provide a more isochronous beam and perhaps higher intensities. Thus for a high-Z experiment, we could consider a configuration similar to Sindrum II $[15,16]$ with forward muons that are deflected through an absorber, which could be tailored to reduce the energy spread onto a stopping target, and which could provide a an intense beam pulse on target with a timing width better matched to the $\sim 80 \mathrm{~ns}$ lifetime of Au-stopped $\mu$ 's

The higher-intensity higher-quality PIP-II beam may also enable a number of other related experiments in the muon campus $(\mu \rightarrow \mathrm{e} \gamma, \mathrm{g}-2$ with low-energy $\mu, \ldots)$ and a redesigned mu2e-II might benefit from synergetic designs.

\section{SUMMARY}

Injection of $800 \mathrm{MeV}$ beam into a mu2e-type production solenoid and target can be obtained. This requires variation of the injection angle and/or positions from those used for $8 \mathrm{GeV}$ beam, and has somewhat different trajectories from that $8 \mathrm{GeV}$ case. Some significant differences in the production solenoid shielding, target geometry, and proton absorber geometry and capacity would be required, and will require detailed study for the mu2e-II LOI.

\section{ACKNOWLEDGMENTS}

I thank D Glenszinski, J. Miller, M. Popovic, V. Pronskikh, T. Roberts, S. Werkema and R. Zwaska for helpful comments and discussion.

\section{REFERENCES}

1. M. Ball et al., The PIP-II Conceptual Design Report (2017).

2. Bartoszek, L. et al., Mu2e Technical Design Report, Mu2e Doc 4299, arXiv 1501.0524.(2015).

3. Mu2e Conceptual Design Report, Mu2e Document 1169-v12 (2012).

4. K. Knoepfel et al., "Feasibility Study for a Next-Generation Mu2e Experiment", arXiv:1307.1168v2 (2013).

5. F. Abusalma et al., "Expression of Interest for Evolution of the Mu2e Experiment", Mu2e docdb-10655, Feb. 18, 2018.

6 A. Vivoli, "PIP-II Transfer Lines Design", Proc. NAPAC 2016, Chicago, IL p. 1161 (2016).

7. W. Chou et al., "8 GeV H- ions: transport and injection," Proc. PAC 2005, Knoxville, TE, p. 1222 (2005).

8. L.R. Scherk, Canadian J. of Phys, 57, 558 (1979).

9. P.B. Keating et al., Phys. Rev. A 52, 4547 (1995).

10. T. Roberts, private communication (2017). 
11. S. Werkema, "High-Priority Accelerator R\&D Needs", presentation at Mu2e-II Workshop, ANL, Argonne, IL, Dec. 8, 2017.

12. V. Pronskikh et al., "Target Station design for the mu2e Experiment", Proc. IPAC2014, Dresden, Deutschland, p. 3970 (2014).

13. H. Wiedemann, "Particle Accelerator Physics", Springer Verlag, (third edition) 2007.

14. V. Pronskikh, D. Glenzinski, K. Knoepfel, N. Mokhov, R. Tschirhart, "A study of the energy dependence of radiation damage in superconducting coils for a next generation Mu2e at PIP-II", FERMILAB-CONF-16-095APC-E, April 2016. arXiv:1612.08931

15. SINDRUM II Collaboration, W. Honecker et al., Phys.Rev.Lett. 76 (1996), 200.

16. W. Bertl et al., European Physics Journal C 47, 337 (2006). 